\title{
Light with Tunable Non-Markovian Phase Imprint
}

\author{
Robert Fischer, ${ }^{1, *}$ Itamar Vidal, ${ }^{2,4}$ Doron Gilboa, ${ }^{4}$ Ricardo R. B. Correia, ${ }^{3}$ Ana C. Ribeiro-Teixeira, ${ }^{3}$ \\ Sandra D. Prado, ${ }^{3}$ Jandir Hickman, ${ }^{3}$ and Yaron Silberberg ${ }^{4}$ \\ ${ }^{1}$ CPGEI, Federal University of Technology-Paraná, 80230-901 Curitiba, PR, Brazil \\ ${ }^{2}$ Grupo de Física Atômica e Lasers, DF-CCEN, Universidade Federal da Paraíba, \\ Cx. Postal 5086, 58051-900 João Pessoa, Paraiba, Brazil \\ ${ }^{3}$ Instituto de Física, Universidade Federal do Rio Grande do Sul, 91501-970 Porto Alegre, RS, Brazil \\ ${ }^{4}$ Department of Physics of Complex Systems, Weizmann Institute of Science, Rehovot 7610001, Israel
}

(Received 7 May 2015; published 14 August 2015)

\begin{abstract}
We introduce a simple and flexible method to generate spatially non-Markovian light with tunable coherence properties in one and two dimensions. The unusual behavior of this light is demonstrated experimentally by probing the far field and by recording its diffraction pattern after a double slit: In both cases we observe, instead of a central intensity maximum, a line- or cross-shaped dark region, whose width and profile depend on the non-Markovian coherence properties. Because these properties can be controlled and easily reproduced in experiment, the presented approach lends itself to serving as a test bed to study and gain a deeper understanding of non-Markovian processes.
\end{abstract}

DOI: 10.1103/PhysRevLett.115.073901

In nature, non-Markovian processes are far more common than Markovian ones; however, scientists often prefer to describe the behavior of nature with Markovian models. This might be the case because Markovian models are easier to build, and it is significantly easier to combine them when constructing more complex models. Recently, however, there has been increasing interest in understanding the specific impact of non-Markovian behavior on chemical and biological processes such as electron transfer [1], the kinetics of protein folding [2], or the ion transport through membranes [3], to name but a few. In optics, the radiation dynamics in photonic crystals [4], optical gain in quantumwell lasers [5], and dephasing processes in coupled quantum-dot cavities [6] have been experimentally observed to be governed by non-Markovian behavior. Characterizing non-Markovian evolutions of quantum properties at the loss of entanglement has become the center of extensive theoretical and experimental efforts $[7,8]$; it is hoped that this will pave the way for better read-out mechanisms in quantum computing [9] or even controlled generation of entangled states [10]. Such quantum phenomena, or their application in logical circuits [11], are often studied using optical means [7,8]. Furthermore, the recent suggestion to initialize controlled quantum states by "optical pumping" [10] opens exciting possibilities for shaping desired quantum properties, including designed non-Markovian characteristics, in the optical regime and then transferring them to other physical systems $[12,13]$.

Non-Markovian light, which might serve as an effective tool towards these objectives, has been previously generated by overlaying a light beam with a delayed copy or echo of itself [14]. An alternative approach employs a micromechanical oscillator as a mirror in order to obtain
PACS numbers: 42.25.Fx, 03.65.Yz, 42.30.Kq, 42.50.Ar

non-Markovian properties from its Brownian movements [15]. The two methods result in light with non-Markovian properties in the time domain; they offer only a limited degree of control and practically no repeatability if the exact same conditions need to be reproduced. In this Letter we, therefore, introduce and experimentally demonstrate a technique to create spatially non-Markovian light to overcome these limitations. By using a spatial light modulator (SLM) to imprint a non-Markovian phase pattern (NMP) onto a coherent light beam, we build an experimental setup that is simple yet flexible and programmable (and, thus, easily reproducible) to generate light with the desired spatial non-Markovian properties in one or two dimensions. Because of this combination of control over the nonMarkovian properties and rapid experimental realization, our approach lends itself to serving as a test bed to gain a deeper understanding of the dynamics of non-Markovian processes, or even as a building block for experiments aiming at producing specific entangled states.

Moreover, NMPs in both one and two dimensions can be generated by algorithms with a tuning parameter that allows us to scan from strictly periodic to non-Markovian properties. Looking at the spatial coherence of the wave front shaped by this means, we observe (depending on the tuning parameter) a short-range anticorrelation and a mid-range correlation, yet practically no correlation beyond a specific distance. Probing this light in two basic experiments, namely, by recording the far field and diffraction on a double slit, we demonstrate how the response of this light differs significantly from the familiar behavior of coherent or thermal light, or any combination of these two.

In a Markovian process, the probability of a given state may depend only upon the one state preceding it (relaxed 
condition), not on the sequence of events that occurred before. Applying the Markov property to the case of a twodimensional matrix, the value at each matrix element should be statistically independent from any other element with the exception of its immediate neighbors on either side. As a consequence, the covariance matrix of a pattern fulfilling the Markov criteria will be (tri)diagonal [16]. However, the Markov condition is violated if the statistical process generating the pattern has some kind of memory. A textbook example would be the drawing of distinct balls from a urn without replacing them, where the probability of a ball to be drawn depends on the outcome of all previous drawings. As shown in Fig. 1(a), we use a slightly modified version of this model to generate one-dimensional NMP: Starting with a random permutation of $L$ symbols (e.g., for $L=9$ the numbers 1-9), the series is continued with a random permutation of its first $s$ elements $(1 \leq s \leq L)$, such that the tuple of the last $L$ elements again forms a permutation of $L$. Then the next $s$ elements in the series are added in randomly permuted order, and the process is repeated until the series reaches the desired length. For our 1D experiments, we combine several such lines (each statistically independent from the others) to a matrix with horizontally non-Markovian, yet vertically Markovian properties.

One convenient method of generating two-dimensional NMPs is the solving of overlapping Latin squares or,

(a) one-dimensional

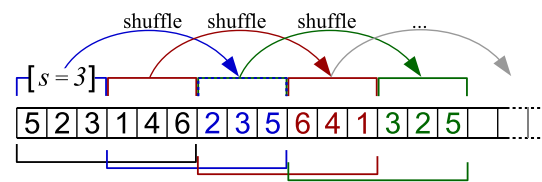

(b)

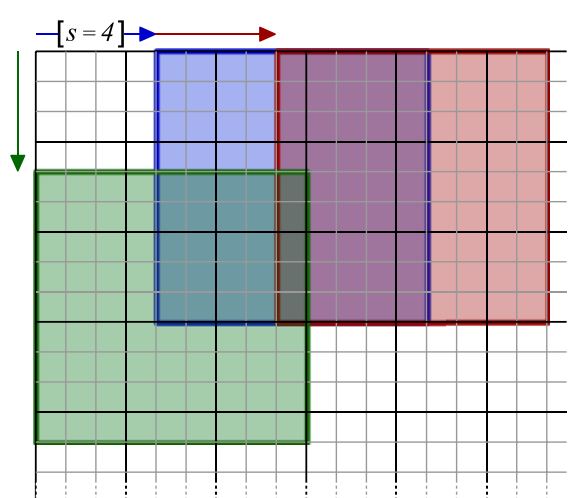

FIG. 1 (color online). Generation of non-Markovian patterns for phase imprint. (a) In one dimension: Generating a series of overlapping permutations (indicated by colored braces below the series) of here $L=6$ symbols with, e.g., a shift size $s=3$. (b) In two dimensions: Solving overlapping Sudoku puzzles after shifting the outer $9 \times 9$ frame by, e.g., $s=4$ elements horizontally or vertically. alternatively, Sudoku puzzles. For this Letter we use common $9 \times 9$ Sudoku puzzles with the numbers $1-9$ as symbols. Starting with one correctly solved Sudoku, we shift its outer frame of size $9 \times 9$ by $s$ elements either horizontally or vertically and fill the empty elements to solve for a valid Sudoku within the new frame position [Fig. 1(b)]. The frame then is scanned in steps of $s$ elements over the matrix until the desired dimensions are reached. The number of $L=9$ symbols was chosen primarily for clarity in the reported figures and the familiarity with regular Sudokus; higher values can be used in both one and two dimensions, which would thus increase the range of $s$ and, equally, the resolution of the transition between strictly period and non-Markovian patterns. In order to emphasize that the use of Sudoku patterns is but one possibility for creating two-dimensional NMPs, we refer in the following to light with such a Sudoku-based non-Markovian phase imprint as "Sudokulight".

To translate the NMP to a phase imprint, each element is assigned a discrete phase level by multiplying the respective number by $2 \pi / L$, and a SLM (Hamamatsu LCOS-SLM $\mathrm{x} 10468)$ is programmed with this phase information. The properties of a plane wave $(\lambda=808 \mathrm{~nm})$ reflected from the SLM surface is then probed in the experimental setup depicted schematically in Fig. 2(a), in which the far field is recorded with a CCD camera.
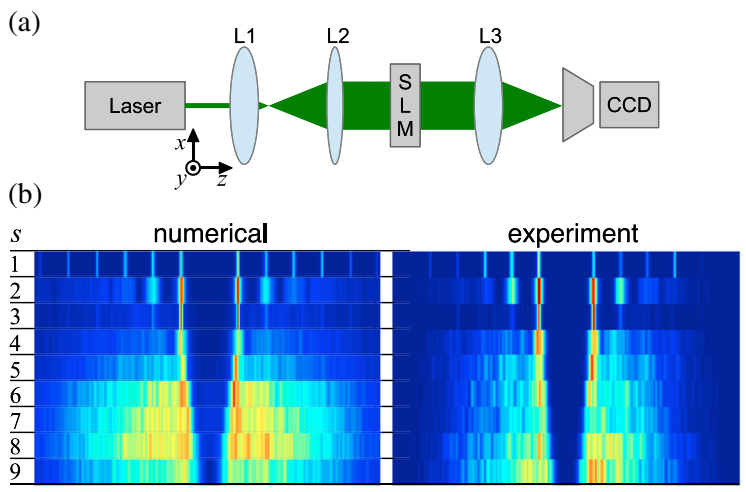

(c) Covariance matrix for $s=2$

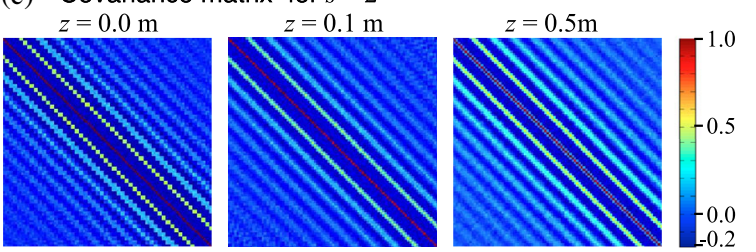

FIG. 2 (color online). Probing the far field of spatially nonMarkovian light. (a) Experimental setup. (b) Numerical (left side) and experimental (right side) results, averaged over multiple realizations, for $1 \mathrm{D}$ non-Markovian phase imprint with 9 equidistant phase levels. Rows depict the $k$-space for $s$ values from 9 (top row) to 1 (bottom row). (c) Central part of covariance matrix $J$ of the complex field for $s=2$ at different propagation distances $z$. 
Applying a 1D NMP at the SLM, we observe in the far field a dark central region [Fig. 2(b)]. Because the phase levels in the experiment are chosen such that the sum of the complex field is zero, a small dark region in the center is to be expected (similar, e.g., to the center of an optical vortex). However, we note that the dip is significantly larger and that its width, edge shape, and depth depend on the parameter $s$ : For $s=1$, which generates strictly periodic patterns, the width reaches from the -1 st to the 1 st diffraction order. The width narrows for increasing $s$ until about half this size for $s=9=L$, which corresponds to a series of random permutations without overlap or "shared" elements. Between these two extremes we observe a transition, where with increasing $s$ the higher diffraction orders (corresponding to higher spatial frequencies) "wash out" and build a relative homogeneous noise floor, which is normally a characteristic of spatially incoherent light. This transition is not linear, as can be seen in Fig. 2(b); in particular, for values of $s$ that are divisors of $L$ (e.g., $s=3$ ) the lower diffraction orders and the central dip appear much more pronounced.

It is important to note that the value of $s$ does not influence the number of occurrences of each phase level (all $L$ phase levels appear equally often), but only restrains to a higher (for smaller $s$ ) or lower (for larger $s$ ) degree the random process determining the sequence in which these phase levels form the pattern. The smaller the value of $s$, the larger the statistical dependence between elements in the pattern. In the case of $s=1$, for instance, the pattern is completely determined by the first $L$ elements, whereas for $s=L$ the pattern consists of statistically independent blocks of permutations with length $L$, wherein each element only depends statistically on its neighbors of the same block. The changes observed in the far field for varying $s$, namely, in the width and shape of the dark region and in the higher spatial frequencies, can therefore be attributed to the respective changes in the non-Markovian properties of the phase imprint.

Using the same parameters as in the numerical simulation shown in Fig. 2(b) (left), we calculated the covariance matrix of the complex field at the SLM surface and after a free-space propagation over distances of 0.1 and $0.5 \mathrm{~m}$ [an exemplar for $s=2$ is illustrated in Fig. 2(c)]. This covariance matrix, also referred to as the complex mutual intensity function, or degree of coherence function [16], reveals the interesting statistics of this light in the $x-y$ plane, i.e., anticorrelation in the immediate short range and periodic peaks of high correlation at midrange, which get weaker at increasing distance from the diagonal (the higher the ratio $s / L$, the faster the peak intensity decays). Interestingly enough, these main features persist even as the beam propagates and the overall degree of coherence increases in accordance with the Gaussian Schell model, causing the broadening of the diagonal for propagation distances of $z=0.1$ and $0.5 \mathrm{~m} \mathrm{[17].}$
A similar behavior can be observed for the 2D case, where the pattern for non-Markovian phase imprint is generated by solving overlapping Sudoku puzzles. As depicted in Fig. 3 (top), the far field features a dark cross, whose width and border shape again depend on the parameter $s$. The transition from structured to relative homogeneous autocorrelation traces of these measurements [Fig. 3 (bottom)] illustrates the decreasing degree of spatial coherence for an increasing value of $s / L$. While the spatial coherence can thus be tuned by the choice of $s$, a comparison with the autocorrelation trace of a uniformly distributed random phase pattern [using the same nine discrete phase levels as the Sudokulight; Fig. 3 (bottom, right column)] makes it clear that even the case $s=L$ is far from being spatially incoherent.

These coherence characteristics also lead to an unusual diffraction pattern of Sudokulight in Young's classical double slit experiment [18]. The fringe contrast at interference is commonly used as a measure for the coherence of light [19]. To test the diffraction of Sudokulight on a double slit, we therefore employ the experimental setup schematically shown in Fig. 4(a). A $4 f$ imaging with a magnification of $1 / 5$ downscales the phase imprint at the plane of the double slit, such that the size of about five elements in the NMP corresponds to the $100-\mu \mathrm{m}$ slit width (slit distance $300 \mu \mathrm{m})$. The diffraction pattern is again recorded with a CCD camera.

While incoherent light produces a Gaussian-shaped intensity diffraction pattern perpendicular to the slit orientation, coherent light produces fringes as seen in Fig. 4(c) (right column). Partially coherent light is known to produce a linear combination of these two patterns, always with an intensity maximum at the center. Sudokulight, on the other hand, features an intensity minimum at the center, not only for the strictly periodic case $s=1$, but for all values of the tuning parameter $s$, both in the one-dimensional [Fig. 4(b)]

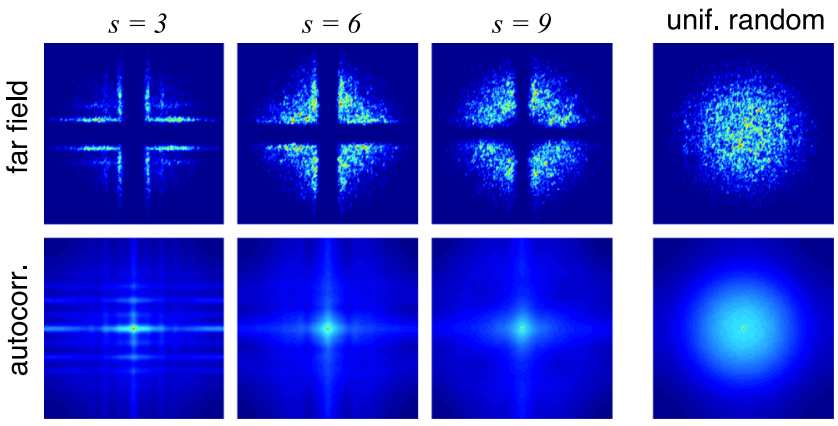

FIG. 3 (color online). Sudokulight with 2D non-Markovian phase imprint. Top row (from left to right) depicts the far field measured with the setup illustrated in Fig. 2(a) for NMPs with $s=3,6$, and 9, respectively, averaged over 100 realizations. Bottom row shows the far-field intensity autocorrelation of the respective measurements. For comparison, the right column shows the respective results of a uniformly distributed random nine-level phase pattern imprinted on the beam. 


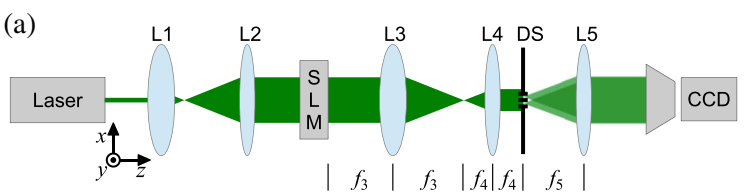

(b)

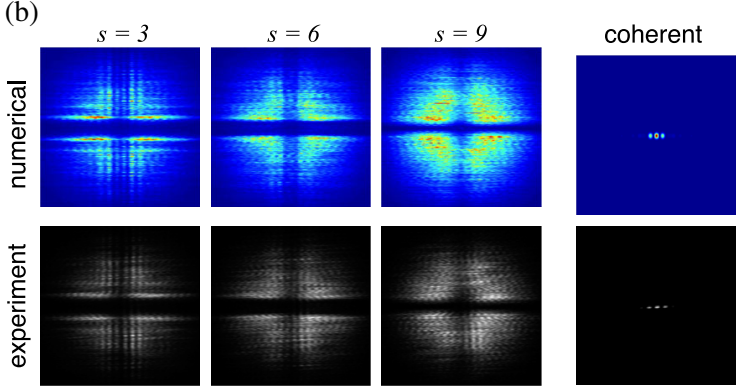

FIG. 4 (color online). Diffraction of Sudokulight on a double slit. All results are averaged over 100 realizations with different NMPs. (a) Experimental setup, employing $4 f$ imaging to reconstruct the phase imprint in the plane of the double slit with a magnification of $1 / 5$. (b) Numerical (left side) and experimental (right side) diffraction patterns for 1D non-Markovian light for increasing parameter $s$. Rows depict the intensity of the diffraction pattern integrated along the slit orientation for $s=1$ (top) to 9 (bottom). (c) Top and bottom rows show the numerical and experimental diffraction patterns obtained for Sudokulight with $s=3,6$, and 9, respectively (slits oriented vertically). The right column depicts the diffraction of a plane wave under the same conditions and scale, showing that the spatial frequency of the interference fringes is the same for coherent and spatially nonMarkovian light.

and the two-dimensional case [Fig. 4(c), columns 1-3, all results averaged over 100 realizations with different NMPs at a fixed value of $s]$. In the two-dimensional case, it is important to distinguish between the two pattern orientations. While the horizontal (perpendicular to the slit orientation) intensity modulation originates from the diffraction on the double slit, the vertical intensity modulation is simply the far field of the light along both slits. By comparing the two orientations in Fig. 4(c), one notes the appearance of additional vertical fringes due to interference of the diffracted light, which vanish for higher values of $s$. This decreasing fringe contrast for an increasing value of $s / L$, which can also be observed in the one-dimensional case, confirms that the spatial coherence can be tuned with the parameter $s$.

We note that the pattern-generation methods used in this Letter are but two possibilities among many to create spatially non-Markovian light via a SLM. To give just one more example, a NMP tiled with nonoverlapping squares (of width $w$ ) that contain a random permutation of numbers 1 to $w^{2}$ produces a donut-shaped intensity pattern in the far field. In contrast to previous approaches that generate nonMarkovian light in time, the spatial analogue therefore offers a far greater freedom and flexibility (along with repeatability) in designing the stochastic and statistical properties of the light. In addition, this approach of using a SLM to imprint a non-Markovian phase can be easily extended to the time domain by rapidly reprogramming the SLM with a series of patterns that depend on their predecessors in a non-Markovian fashion. Different nonMarkovian behaviors observed in natural systems can thus be simulated more accurately with the corresponding pattern-generation method. Although any rule that correlates a matrix element beyond its immediate neighbor may produce a NMP, methods with a tuning parameter, such as the shift size $s$, allow for a deeper understanding of the impact of non-Markovian properties on the system of interest.

In conclusion, the generation of spatially non-Markovian light with tunable properties, as demonstrated in this Letter for one and two dimensions, lends itself to serving as test bed for the study of non-Markovian systems and dynamics. Light with a non-Markovian phase imprint shows very unusual behavior even in classical linear experiments, such as the diffraction on a double slit. Further research is needed to show how this light interacts with nonlinear systems. As it maintains its particular spatial coherence properties when propagating in free space, this light might well serve as a building block to designing specific, nonMarkovian properties in the optical regime, which could then be transferred to other physical systems [12,13]. Because of the flexibility in controlling the spatial coherence with the suggested pattern-generation method and the easy, fast, and reproducible experimental implementation via a spatial light modulator, the approach presented in this Letter may become a useful tool in the search for a deeper understanding of non-Markovian processes.

This work was supported by grants from the CAPESWeizmann cooperation program and $\mathrm{CNPq}$ (Universal Grant No. 483983/2013-6).

*Corresponding author. $\mathrm{r} @$ robertfischer.eu

[1] R.-X. Xu, Y. Chen, P. Cui, H.-W. Ke, and Y. Yan, The quantum solvation, adiabatic versus nonadiabatic, and Markovian versus non-Markovian nature of electrontransfer rate processes, J. Phys. Chem. A 111, 9618 (2007).

[2] S. S. Plotkin and P. G. Wolynes, Non-Markovian Configurational Diffusion and Reaction Coordinates for Protein Folding, Phys. Rev. Lett. 80, 5015 (1998).

[3] I. Goychuk and P. Hänggi, Non-Markovian Stochastic Resonance, Phys. Rev. Lett. 91, 070601 (2003).

[4] U. Hoeppe, C. Wolff, J. Küchenmeister, J. Niegemann, M. Drescher, H. Benner, and K. Busch, Direct Observation of non-Markovian Radiation Dynamics in 3D Bulk Photonic Crystals, Phys. Rev. Lett. 108, 043603 (2012).

[5] D. Ahn, Theory of non-Markovian optical gain in quantumwell lasers, Prog. Quantum Electron. 21, 249 (1997).

[6] P. Kaer, T. R. Nielsen, P. Lodahl, A.-P. Jauho, and J. Mørk, Non-Markovian Model of Photon-Assisted Dephasing by 
Electron-Phonon Interactions in a Coupled Quantum-DotCavity System, Phys. Rev. Lett. 104, 157401 (2010).

[7] A. Chiuri, C. Greganti, L. Mazzola, M. Paternostro, and P. Mataloni, Linear optics simulation of quantum nonMarkovian dynamics, Sci. Rep. 2, 968 (2012).

[8] B.-H. Liu, L. Li, Y.-F. Huang, C.-F. Li, G.-C. Guo, E.-M. Laine, H.-P. Breuer, and J. Piilo, Experimental control of the transition from Markovian to non-Markovian dynamics of open quantum systems, Nat. Phys. 7, 931 (2011).

[9] G. Goldstein, P. Cappellaro, J. R. Maze, J. S. Hodges, L. Jiang, A. S. Sørensen, and M. D. Lukin, EnvironmentAssisted Precision Measurement, Phys. Rev. Lett. 106, 140502 (2011).

[10] J. Cho, S. Bose, and M. S. Kim, Optical Pumping into Many-Body Entanglement, Phys. Rev. Lett. 106, 020504 (2011).

[11] A. Crespi, R. Ramponi, R. Osellame, L. Sansoni, I. Bongioanni, F. Sciarrino, G. Vallone, and P. Mataloni, Integrated photonic quantum gates for polarization qubits, Nat. Commun. 2, 566 (2011).

[12] J. T. Barreiro, M. Müller, P. Schindler, D. Nigg, T. Monz, M. Chwalla, M. Hennrich, C. F. Roos, P. Zoller, and R. Blatt,
An open-system quantum simulator with trapped ions, Nature (London) 470, 486 (2011).

[13] M. Paternostro, D. Vitali, S. Gigan, M. S. Kim, C. Brukner, J. Eisert, and M. Aspelmeyer, Creating and Probing Multipartite Macroscopic Entanglement with Light, Phys. Rev. Lett. 99, 250401 (2007).

[14] R. Beach and S. R. Hartmann, Incoherent Photon Echoes, Phys. Rev. Lett. 53, 663 (1984).

[15] S. Groeblacher, A. Trubarov, N. Prigge, M. Aspelmeyer, and J. Eisert, Observation of non-Markovian micro-mechanical Brownian motion, arXiv:1305.6942.

[16] R. Horstmeyer, R. Y. Chen, B. Judkewitz, and C. Yang, Markov speckle for efficient random bit generation, Opt. Express 20, 26394 (2012).

[17] L. Mandel and E. Wolf, Optical Coherence and Quantum Optics (Cambridge University Press, Cambridge, England, 1995).

[18] T. Young, The Bakerian lecture: Experiments and calculations relative to physical optics, Phil. Trans. R. Soc. London 94, 1 (1804).

[19] J. W. Goodman, Statistical Optics (Wiley, New York, 2000). 